\title{
Analytical solution of non-linear fractional diffusion equation
}

\section{Obaid Alqahtani ${ }^{1 *}$ (1)}

"Correspondence: OBALGAHTANI@ksu.edu.sa

${ }^{1}$ Department of Mathematics, King Saud University, Riyadh 11451 Saudi Arabia

\section{Springer}

\begin{abstract}
In this paper, we obtain an approximate/analytical solution of nonlinear fractional diffusion equation using the q-homotopy analysis transform method. The existence and uniqueness of the solution for this problem are also derived. Further, the applicability of the model is discussed based on graphical results and numerical examples.
\end{abstract}

MSC: 26A33; 33E12; 35A22; 35R11

Keywords: Fractional diffusion equation; Existence and Uniqueness; Homotopy Perturbation method

\section{Introduction}

Many phenomena of the physical sciences are associated with the idea of diffusion like populations of different kinds diffuse; particles in a solvent and other substances diffusing; ions diffusing and electrons; and the momentum of a viscous fluid diffusing. The older work concerns the description of mass diffusion and heat propagation by means of partial differential equations (PDEs). The type of PDEs used is the so-called parabolic equations, a family based on the properties of the most classical model, the linear heat equation, which is called in this context the diffusion equation (see [1]). Nonlinear diffusion equations are a generalization of diffusion and it comes from a variety of diffusion phenomena which appear widely in nature. The detailed derivation of the nonlinear diffusion equation can be found in [2].

Presently, fractional differential equations play an important role in various fields like engineering, biology, special functions, geophysics, acoustic dissipation, biomedical, signal and image processing, control theory, integral representations and singularities analysis, special functions, viscoelasticity, non-Fourier heat conduction, electricity, mechanics and fluid dynamics. To study fractional calculus, a lot of material is freely available. For more details about fractional calculus and its applications see [3-19].

\section{Atangana-Baleanu fractional derivative}

In 2016, Atangana and Baleanu [20] suggested a fractional derivative operator based on the well-known generalized Mittag-Leffler function. Many researchers have applied this

(c) The Author(s) 2021. This article is licensed under a Creative Commons Attribution 4.0 International License, which permits use sharing, adaptation, distribution and reproduction in any medium or format, as long as you give appropriate credit to the original author(s) and the source, provide a link to the Creative Commons licence, and indicate if changes were made. The images or other third party material in this article are included in the article's Creative Commons licence, unless indicated otherwise in a credit line to the material. If material is not included in the article's Creative Commons licence and your intended use is not permitted by statutory regulation or exceeds the permitted use, you will need to obtain permission directly from the copyright holder. To view a copy of this licence, visit http://creativecommons.org/licenses/by/4.0/. 
operator in various mathematical model, for example, Chua's circuit model [21], RC, LC, and RL electircal circuits [22], the Cattaneo-Hristov model of elastic heat diffusion, and the Casson fluid model with heat generation and chemical reaction [23]. In this section, we briefly review the fractional order Atangana-Baleanu derivative (AB-derivative) and its properties.

Definition 2.1 ([24]) Consider $g \in H^{1}[a, b], a<b$, then the Caputo sense AB-derivative is defined as

$$
{ }_{a}^{A B C} D_{t}^{\alpha} g(t)=\frac{N(\alpha)}{1-\alpha} \int_{a}^{t} g^{\prime}(\theta) E_{\alpha}\left[-\alpha \frac{(t-\theta)^{\alpha}}{1-\alpha}\right] d \theta, \quad \alpha \in[0,1]
$$

where $E_{\alpha}$ is the Mittag-Leffler function and $N(\alpha)$ is a normalization function with $N(0)=$ $N(1)=1$.

We now consider the Laplace transform for the fractional differential operator for $n=1$.

Definition 2.2 ([24]) Consider $g$ is a function continuous on $[a, b]$, the Laplace transform for AB-derivative is defined as

$$
L\left\{_{0}^{A B C} D_{t}^{\alpha} g(t)\right\}(p)=\frac{\mathcal{B}(\alpha)}{1-\alpha} \frac{p^{\alpha} L\{g(t)\}(p)-p^{\alpha-1} g(0)}{p^{\alpha}+\frac{\alpha}{1-\alpha}},
$$

where $0<\alpha \leq 1$.

Theorem 2.1 ([25]) Consider $g$ is a function continuous on $[a, b]$, then

$$
\left\|_{0}^{A B R} D_{t}^{\alpha} g(t)\right\|<\frac{\mathcal{B}(\alpha)}{1-\alpha}\|g(x)\|,
$$

where $0<\alpha<1$.

Theorem 2.2 ([25]) The Atangana-Baleanu Caputo fractional derivative operator satisfies the Lipschitz condition

$$
\left\|_{0}^{A B C} D_{t}^{\alpha} f(t)-{ }_{0}^{A B C} D_{t}^{\alpha} g(t)\right\| \leq H\|f(t)-g(t)\| .
$$

Theorem 2.3 ([25]) For the time-fractional ordinary differential equation

$$
{ }_{0}^{A B C} D_{t}^{\alpha} f(t)=\mathcal{U}(t),
$$

the unique solution is given by using the Laplace transform

$$
f(t)=\frac{1-\alpha}{\mathcal{B}(\alpha) \mathcal{U}(t)}+\frac{\alpha}{\mathcal{B}(\alpha) \Gamma(\alpha)} \int_{a}^{t} \frac{\mathcal{U}(y)}{(t-y)^{1-\alpha}} d y
$$

The detailed proof of the above theorems can be found in [26]. 
Remark From the above theorem, the AB-fractional integral is the average between the given function and the function's Riemann-Liouville fractional integral. Hence this is more general than the Riemann-Liouville fractional derivative as well as the Caputo operator. Also from the definition of the Atangana-Baleanu operator, it is clear that if $\alpha=0$ is zero, we obtain the initial function and if $\alpha$ is 1 , we get the ordinary integral.

Definition 2.3 ([24]) The fractional integral of order $\alpha$ is defined as

$$
{ }_{a}^{A B} I_{t}^{\alpha} g(t)=\frac{1-\alpha}{\mathcal{B}(\alpha)} g(t)+\frac{\alpha}{\mathcal{B}(\alpha) \Gamma(\alpha)} \int_{a}^{t} g(y)(t-y)^{\alpha-1} d y .
$$

If we take $\alpha=0$, we get the initial function and taking $\alpha=1$ we obtain the classical integral of order one. In a recent paper of Tetashi et al. [27], they compared the model of anomalous diffusion using the Riemann-Liouville [3], Caputo-Fabrizio [8] and AB-derivative [26]. By analyzing the corresponding fractional diffusion equations within the continuous time random walk framework, they obtained waiting time distributions characterized by exponential, stretched exponential, and power-law functions, as well as a crossover between two behaviors. Further, the authors discussed the effect of non-singular kernel $K(t)=\frac{\mathcal{B}(\alpha)}{1-\alpha} E_{\alpha}\left[-\alpha \frac{t^{\alpha}}{1-\alpha}\right]$ using the solution of anomalous diffusion.

In this paper, we will extend an idea for fractional diffusion equation using the AtanganaBaleanu operator to nonlinear fractional diffusion model by considering the following equation:

$$
{ }_{0}^{A B C} D_{t}^{\alpha} \mathcal{U}(x, t)=\frac{\partial}{\partial x}\left(\mathcal{U}^{a} \frac{\partial \mathcal{U}(x, t)}{\partial x}\right)
$$

with

$$
\mathcal{U}(x, 0)=\phi(x)
$$

Our main goal of this paper is to solve Eq. (2.8) under the condition (2.9). In Sect. 3, we start with existence and uniqueness of the solution of nonlinear fractional diffusion equation. Section 4 is devoted to finding an approximate solution of Eq. (2.8) using a $q$-homotopy analysis method and in Sect. 5, we discuss the special cases of (2.8) by taking $a=0, a>0$ and $a<0$.

\section{Existence of solution for the diffusion equation model}

Assume that the function $f\left(x, t, \mathcal{U}, \mathcal{U}^{\prime}, \mathcal{U}^{\prime \prime}\right)$ satisfies the Lipschitz condition

$$
\begin{aligned}
& \left\|f\left(x, t, \mathcal{U}, \mathcal{U}^{\prime}, \mathcal{U}^{\prime \prime}\right)-f\left(x, t, \mathcal{U}_{1}, \mathcal{U}_{1}^{\prime}(t), \mathcal{U}_{1}^{\prime \prime}(t)\right)\right\| \\
& \quad \leq M\left|\mathcal{U}-\mathcal{U}_{1}\right|+K\left|\mathcal{U}^{\prime}-\mathcal{U}_{1}^{\prime}(t)\right|+L\left|\mathcal{U}^{\prime \prime}-\mathcal{U}_{1}^{\prime \prime}(t)\right|
\end{aligned}
$$

Further, assume that

$$
\begin{aligned}
& \left\|\mathcal{U}^{\prime}-\mathcal{U}_{1}^{\prime}(t)\right\| \leq \delta_{1}\left\|\mathcal{U}-\mathcal{U}_{1}\right\|, \\
& \left\|\mathcal{U}^{\prime \prime}-\mathcal{U}_{1}^{\prime \prime}(t)\right\| \leq \delta_{2}\left\|\mathcal{U}-\mathcal{U}_{1}\right\|,
\end{aligned}
$$


where

$$
\delta_{1}, \delta_{2} \in \mathbb{R}^{+}
$$

such that $M+K \delta_{1}+L \delta_{2} \leq 1$; then the solution for the time-fractional differential equation

$$
{ }_{0}^{A B C} D_{t}^{\alpha} \mathcal{U}(x, t)=f\left(x, t, \mathcal{U}, \mathcal{U}^{\prime}, \mathcal{U}^{\prime \prime}\right)
$$

exists if we are able to find $t_{\max }$ such that

$$
\left(\frac{1-\alpha}{\mathcal{B}(\alpha)}+\frac{t_{\max }^{\alpha}}{\mathcal{B}(\alpha) \Gamma(\alpha)}\right)<1
$$

Proof Using the fundamental theorem of fractional calculus, Eq. (3.1) can be written as

$$
\begin{aligned}
\mathcal{U}(x, t)-\mathcal{U}(x, 0)= & \frac{1-\alpha}{\mathcal{B}(\alpha)} f\left(x, t, \mathcal{U}, \mathcal{U}^{\prime}, \mathcal{U}^{\prime \prime}\right) \\
& +\frac{\alpha}{\mathcal{B}(\alpha) \Gamma(\alpha)} \int_{0}^{t}(t-y)^{\alpha-1} f\left(x, t, \mathcal{U}, \mathcal{U}^{\prime}, \mathcal{U}^{\prime \prime}\right) d y .
\end{aligned}
$$

We now present the recursive formula

$$
\begin{aligned}
\mathcal{U}_{n}(x, t)= & \frac{1-\alpha}{\mathcal{B}(\alpha)} f\left(x, t, \mathcal{U}_{n-1}, \mathcal{U}_{n-1}^{\prime}, \mathcal{U}_{n-1}^{\prime \prime}\right) \\
& +\frac{\alpha}{\mathcal{B}(\alpha) \Gamma(\alpha)} \int_{0}^{t}(t-y)^{\alpha-1} f\left(x, t, \mathcal{U}_{n-1}, \mathcal{U}_{n-1}^{\prime}, \mathcal{U}_{n-1}^{\prime \prime}\right) d y
\end{aligned}
$$

Take the difference of consecutive terms $\gamma_{n}=\mathcal{U}_{n}-\mathcal{U}_{n-1}$.

This gives

$$
\begin{aligned}
\gamma_{n}= & \frac{1-\alpha}{\mathcal{B}(\alpha)}\left(f\left(x, t, \mathcal{U}_{n-1}, \mathcal{U}^{\prime}{ }_{n-1}, \mathcal{U}^{\prime \prime}{ }_{n-1}\right)\right. \\
& \left.-f\left(x, t, \mathcal{U}_{n-2}, \mathcal{U}^{\prime}{ }_{n-2}, \mathcal{U}^{\prime \prime}{ }_{n-2}\right)\right) \\
& +\frac{\alpha}{\mathcal{B}(\alpha) \Gamma(\alpha)} \int_{0}^{t}(t-y)^{\alpha-1}\left(f\left(y, t, \mathcal{U}_{n-1}, \mathcal{U}^{\prime}{ }_{n-1}, \mathcal{U}^{\prime \prime}{ }_{n-1}\right)\right. \\
& \left.-f\left(y, t, \mathcal{U}_{n-2}, \mathcal{U}^{\prime}{ }_{n-2}, \mathcal{U}^{\prime \prime}{ }_{n-2}\right)\right) d y .
\end{aligned}
$$

We observe that

$$
\mathcal{U}_{n}=\sum_{i=1}^{n} \gamma_{i}
$$

taking the norm of (3.4), and using the well-known triangular inequality, we get

$$
\begin{aligned}
\left\|\gamma_{n}\right\|= & \left\|\mathcal{U}_{n}-\mathcal{U}_{n-1}\right\| \\
\leq & \frac{1-\alpha}{\mathcal{B}(\alpha)} \| f\left(x, t, \mathcal{U}_{n-1}, \mathcal{U}^{\prime}{ }_{n-1}, \mathcal{U}^{\prime \prime}{ }_{n-1}\right) \\
& -f\left(x, t, \mathcal{U}_{n-2}, \mathcal{U}^{\prime}{ }_{n-2}, \mathcal{U}^{\prime \prime}{ }_{n-2}\right) \|
\end{aligned}
$$




$$
\begin{aligned}
& +\frac{\alpha}{\mathcal{B}(\alpha) \Gamma(\alpha)} \| \int_{0}^{t}(t-y)^{\alpha-1}\left(f\left(y, t, \mathcal{U}_{n-1}, \mathcal{U}^{\prime}{ }_{n-1}, \mathcal{U}^{\prime \prime}{ }_{n-1}\right)\right. \\
& \left.-f\left(y, t, \mathcal{U}_{n-2}, \mathcal{U}^{\prime}{ }_{n-2}, \mathcal{U}^{\prime \prime}{ }_{n-2}\right)\right) d y \|
\end{aligned}
$$

Now, use the fact that $f\left(x, t, \mathcal{U}, \mathcal{U}^{\prime}, \mathcal{U}^{\prime \prime}\right)$ satisfies the Lipschitz condition, defined as

$$
\begin{aligned}
& \left\|f\left(x, t, \mathcal{U}, \mathcal{U}^{\prime}, \mathcal{U}^{\prime \prime}\right)-f\left(x, t, u_{1}, \mathcal{U}_{1}^{\prime}(t), \mathcal{U}_{1}^{\prime \prime}(t)\right)\right\| \\
& \quad \leq M\left|\mathcal{U}-\mathcal{U}_{1}\right|+K\left|\mathcal{U}^{\prime}-\mathcal{U}_{1}^{\prime}(t)\right|+L\left|\mathcal{U}_{1}^{\prime \prime}(t)-\mathcal{U}_{1}^{\prime \prime}(t)\right| .
\end{aligned}
$$

We have

$$
\begin{aligned}
\left\|\gamma_{n}\right\| \leq & \frac{1-\alpha}{\mathcal{B}(\alpha)}\left[M\left\|\mathcal{U}_{n-1}-\mathcal{U}_{n-2}\right\|\right. \\
& \left.+K\left\|\mathcal{U}_{n-1}^{\prime}-\mathcal{U}_{n-2}^{\prime}\right\|+L\left\|\mathcal{U}_{n-1}^{\prime \prime}-\mathcal{U}_{n-2}^{\prime \prime}\right\|\right] \\
& +\frac{\alpha}{\mathcal{B}(\alpha) \Gamma(\alpha)}\left[M\left\|\mathcal{U}_{n-1}-\mathcal{U}_{n-2}\right\|\right. \\
& \left.+K\left\|\mathcal{U}_{n-1}^{\prime}-\mathcal{U}_{n-2}^{\prime}\right\|+L\left\|\mathcal{U}_{n-1}^{\prime \prime}-\mathcal{U}_{n-2}^{\prime \prime}\right\|\right] \int_{0}^{t}(t-y)^{\alpha-1} d y
\end{aligned}
$$

It gives

$$
\begin{aligned}
& {\left[M\left\|\mathcal{U}_{n-1}-\mathcal{U}_{n-2}\right\|+K\left\|\mathcal{U}_{n-1}^{\prime}-\mathcal{U}_{n-2}^{\prime}\right\|+L\left\|\mathcal{U}_{n-1}^{\prime \prime}-\mathcal{U}_{n-2}^{\prime \prime}\right\|\right]} \\
& \quad \times\left[\frac{1-\alpha}{\mathcal{B}(\alpha)}+\frac{\alpha}{\mathcal{B}(\alpha) \Gamma(\alpha)} \int_{0}^{t}(t-y)^{\alpha-1} d y\right]
\end{aligned}
$$

using $\gamma_{n}=\mathcal{U}_{n}-\mathcal{U}_{n-1}$, we have $\gamma_{n-1}=\mathcal{U}_{n-1}-\mathcal{U}_{n-2}$ and assuming

$$
\begin{aligned}
& \left\|\gamma_{n-1}^{\prime}\right\| \leq \delta_{1}\left\|\gamma_{n-1}\right\|, \\
& \left\|\gamma_{n-1}^{\prime \prime}\right\| \leq \delta_{2}\left\|\gamma_{n-1}\right\|,
\end{aligned}
$$

where

$$
\delta_{1}, \delta_{2} \in \mathbb{R}
$$

gives

$$
\begin{aligned}
\left\|\gamma_{n}\right\| \leq & \left(M\left\|\gamma_{n-1}\right\|+K \delta_{1}\left\|\gamma_{n-1}\right\|+L \delta_{2}\left\|\gamma_{n-1}\right\|\right) \\
& \times\left[\frac{1-\alpha}{\mathcal{B}(\alpha)}+\frac{\alpha}{\mathcal{B}(\alpha) \Gamma(\alpha)} \int_{0}^{t}(t-y)^{\alpha-1} d y\right] \\
= & \left\|\gamma_{n-1}\right\|\left(M+K \delta_{1}+L \delta_{2}\right)\left[\frac{1-\alpha}{\mathcal{B}(\alpha)}+\frac{\alpha}{\mathcal{B}(\alpha) \Gamma(\alpha)} \int_{0}^{t}(t-y)^{\alpha-1} d y\right] .
\end{aligned}
$$

Finally, we have

$$
\left\|\gamma_{n}\right\| \leq\left(\frac{1-\alpha}{\mathcal{B}(\alpha)}+\frac{\alpha}{\mathcal{B}(\alpha) \Gamma(\alpha)} \int_{0}^{t}(t-y)^{\alpha-1} d y\right)^{n}\left(M+K \delta_{1}+L \delta_{2}\right)^{n}\left\|\gamma_{0}\right\| .
$$


Now let us consider

$$
M+K \delta_{1}+L \delta_{2}<1
$$

and let

$$
M+K \delta_{1}+L \delta_{2}=\delta_{3}
$$

Then we have

$$
\left\|\gamma_{n}\right\|=\delta_{3}^{n}\left\|\gamma_{0}\right\|\left(\frac{1-\alpha}{\mathcal{B}(\alpha)}+\frac{t_{\max }^{\alpha}}{\mathcal{B}(\alpha) \Gamma(\alpha)}\right)^{n}
$$

The fractional differential equation (2.8) has a solution, which is unique, if we are able to find $t_{\max }$ such that

$$
\left(\frac{1-\alpha}{\mathcal{B}(\alpha)}+\frac{t_{\max }^{\alpha}}{\mathcal{B}(\alpha) \Gamma(\alpha)}\right) \delta_{3}<1
$$

\section{Uniqueness of the solution}

In this section we will show that the above defined problem has a unique solution.

If possible, let $\mathcal{U}(t)$ and $\mathcal{U}_{1}(t)$ be two solutions of the problem (3.1). Consider

$$
\begin{aligned}
\mathcal{U}(x, t)-\mathcal{U}_{1}(x, t)= & \frac{1-\alpha}{\mathcal{B}(\alpha)}\left[f(t, \mathcal{U})-f\left(t, \mathcal{U}_{1}\right)\right] \\
& +\frac{\alpha}{\mathcal{B}(\alpha) \Gamma(\alpha)} \int_{0}^{t}(t-y)^{\alpha-1}\left[f(s, \mathcal{U})-f\left(s, \mathcal{U}_{1}\right)\right] d s
\end{aligned}
$$

applying the norm to both sides of Eq. (4.1),

$$
\begin{aligned}
\mathcal{U}(x, t)-\mathcal{U}_{1}(x, t) \leq & \frac{1-\alpha}{\mathcal{B}(\alpha)}\left\|f(t, \mathcal{U})-f\left(t, \mathcal{U}_{1}\right)\right\| \\
& +\frac{\alpha}{\mathcal{B}(\alpha) \Gamma(\alpha)} \int_{0}^{t} \|(t-y)^{\alpha-1}\left[f(s, \mathcal{U})-f\left(s, \mathcal{U}_{1}\right) \| d s\right.
\end{aligned}
$$

Using the Lipchitz condition and keeping in mind that the obtained result is bounded, we obtain $\mathcal{U}(t)=\mathcal{U}_{1}(t)$. This shows the uniqueness of the solution for Eq. (3.1)

Remark On taking $f\left(x, t, \mathcal{U}, \mathcal{U}^{\prime}, \mathcal{U}^{\prime \prime}\right)$ as $\frac{\partial}{\partial x}\left(\mathcal{U}^{a} \frac{\partial \mathcal{U}(x, t)}{\partial x}\right)$ and following the same assumptions, the solution for the time-fractional diffusion equation

$$
\begin{gathered}
{ }_{0}^{A B C} D_{t}^{\alpha} \mathcal{U}(x, t)=\frac{\partial}{\partial x}\left(\mathcal{U}^{a} \frac{\partial \mathcal{U}(x, t)}{\partial x}\right), \\
0<\alpha \leq 1, a \in \mathbb{R}, 0 \leq x, t \leq 1,
\end{gathered}
$$

exists and is unique. 


\section{5 q-Homotopy analysis transform method}

Perturbation techniques are widely used to find an approximate solution of nonlinear differential equations. In this technique, we use perturbation quantities to create infinitely many linear sub-problems and using a solution of these sub-problems, we obtain the approximate solution in the form of an infinite series. But it is difficult to find an approximate analytical solution for the nonlinear problems having strong nonlinear quantities. This indicates that perturbation approximations are only valid for nonlinear problems having weak nonlinearity. To overcome this, a kind of analytic technique namely a homotopy analysis method was proposed by means of homotopy (a concept of topology). The homotopy analysis method (HAM) $[28,29]$ is one of the best methods to obtain the solution of linear as well as nonlinear differential equations. In HAM, we have $h$ as an auxiliary parameter, which is useful to adjust and control the convergence of some series solution. In 2012, El-Tawil and Huseen [30] proposed the $q$-homotopy analysis method ( $q$-HAM), which is a generalization of the HAM. The essential idea of this method is to introduce a homotopy parameter, say $q$, which varies from 0 to $1 / n$, and a nonzero auxiliary parameter $h$. The $q$-HAM method is more useful than HAM because $q$-HAM provides us with auxiliary parameters $h$ and $n$ used to adjust and control the convergence of series solution (see [31, 32]).

We will apply the $q$-homotopy Laplace transform method to solving (2.8) and (2.9). On taking the Laplace transform of (2.8) and using the initial conditions

$$
\mathcal{U}(x, 0)=\phi(x)
$$

we obtain

$$
\frac{\mathcal{B}(\alpha)}{1-\alpha} \frac{p^{\alpha} L\{\mathcal{U}(x, t)\}(p)-p^{\alpha-1} \mathcal{U}(x, 0)}{p^{\alpha}+\frac{\alpha}{1-\alpha}}=L\left\{f\left(\mathcal{U}, \mathcal{U}^{\prime}, \mathcal{U}^{\prime \prime}\right)\right\}
$$

where $f\left(t, \mathcal{U}, \mathcal{U}^{\prime}, \mathcal{U}^{\prime \prime}\right)=\frac{\partial}{\partial x}\left(\mathcal{U}^{a} \frac{\partial \mathcal{U}(x, t)}{\partial x}\right),=a \mathcal{U}^{a-1}\left(\frac{\partial \mathcal{U}}{\partial x}\right)^{2}+\mathcal{U}^{a} \frac{\partial^{2} \mathcal{U}}{\partial x^{2}}$; then a simple calculation yields

$$
\begin{aligned}
L\{\mathcal{U}(x, t)\}= & \frac{p^{\alpha-1} \mathcal{U}(x, 0)}{p^{\alpha}}+\frac{(1-\alpha)}{\mathcal{B}(\alpha) p^{\alpha}}\left(p^{\alpha}+\frac{\alpha}{1-\alpha}\right) \\
& \times L\left\{a \mathcal{U}^{a-1}\left(\frac{\partial \mathcal{U}}{\partial x}\right)^{2}+\mathcal{U}^{a} \frac{\partial^{2} \mathcal{U}}{\partial x^{2}}\right\} .
\end{aligned}
$$

We have the nonlinear operator

$$
\begin{aligned}
& N[\sigma(x, t: q)] \\
& =\left(\frac{p^{\alpha-1} \mathcal{U}(x, 0)}{p^{\alpha}}\right)+\left[\frac{(1-\alpha)}{\mathcal{B}(\alpha) p^{\alpha}}\left(p^{\alpha}+\frac{\alpha}{1-\alpha}\right)\right] \\
& \quad \times\left(L\left\{a(\sigma(x, t: q))^{a-1}\left(\frac{\partial \sigma(x, t: q)}{\partial x}\right)^{2}+(\sigma(x, t: q))^{a} \frac{\partial^{2} \sigma(x, t: q)}{\partial x^{2}}\right\}\right),
\end{aligned}
$$

where $q \in\left[0, \frac{1}{n}\right], n \geq 1$ is known as an embedding parameter. To solve our problem we will use q-HATM [32] to construct the homotopy in such a way that

$$
(1-n q) L\left\{\sigma(x, t: q)-\mathcal{U}_{0}(x, t)\right\}=h q N[\sigma(x, t: q)]
$$


where $\mathcal{U}_{0}(x, t)$ is the initial value of $\mathcal{U}(x, t), \sigma(x, t)$ is an unknown function and $h \neq 0$ is an auxiliary parameter.

Now, on taking $q=0$ and $q=1 / n$, we obtain from Eq. (5.5)

$$
\sigma(x, t: 0)=\mathcal{U}_{0}(x, t), \quad \rho\left(x, t ; \frac{1}{n}\right)=\mathcal{U}(x, t) .
$$

From Eq. (5.6), we see that, on increasing q from 0 to $1 / n$, our solution $\sigma(x, t: q)$ moves from the initial solution $\mathcal{U}_{0}(x, t)$ to the exact solution $\mathcal{U}(x, t)$.

On expanding $\sigma(x, t: q)$ by a Taylor series, we get

$$
\rho(x, t ; q)=\mathcal{U}_{0}(x, t)+\sum_{m=1}^{\infty} \mathcal{U}_{m}(x, t) q^{m},
$$

where

$$
\mathcal{U}_{m}(x, t)=\left.\frac{1}{m !} \frac{\partial^{m} \rho(x, t ; q)}{\partial q^{m}}\right|_{q=0} .
$$

If we choose $\mathcal{U}_{0}(x, t), n$, and $h$, in a perfect manner, Eq. (5.7) converges at $q=1 / n$,

$$
\mathcal{U}(x, t)=\mathcal{U}_{0}(x, t)+\sum_{m=1}^{\infty} \mathcal{U}_{m}(x, t)\left(\frac{1}{n}\right)^{m}
$$

the result defined in (5.9) is the solution of (2.8) Defining the vectors $\overline{\mathcal{U}}_{r}=\left\{\mathcal{U}_{0}, \mathcal{U}_{1}, \ldots, \mathcal{U}_{r}\right\}$, differentiating $m$ times of Eq. (5.5) w.r.t. q, and then substituting $q=0$, and lastly dividing by $m !$, we get

$$
L\left[\mathcal{U}_{m}(x, t)-k_{m} \mathcal{U}_{m-1}(x, t)\right]=h \Re_{m}\left(\mathcal{U}_{m-1}(x, t)\right),
$$

where

$$
\Re_{m}\left(\mathcal{U}_{m}(x, t)=\left.\frac{1}{(m-1) !} \frac{\partial^{m-1} N(x, t ; q)}{\partial q^{m-1}}\right|_{q=0}\right.
$$

and

$$
k_{m}= \begin{cases}0, & m \leq 1, \\ n, & \text { otherwise } .\end{cases}
$$

Applying the inverse Laplace transform to Eq. (5.10), we have

$$
\mathcal{U}_{m}(x, t)=k_{m} \mathcal{U}_{m-1}(x, t)+h L^{-1}\left[\Re_{m}\left(\mathcal{U}_{m-1}\right)\right] .
$$

With the help of the initial condition which is given in Eq. (2.9) and using Eq. (5.13), we obtain

$$
\mathcal{U}(x, t)=\lim _{n \rightarrow \infty} \sum_{m=0}^{\infty} \mathcal{U}_{m}(x, t)\left(\frac{1}{n}\right)^{m} .
$$

Due to the factor $(1 / n)^{m}$, it gives speedy convergence to the exact solution. 


\section{Special cases to the nonlinear diffusion equation}

By substituting various values of $a$, we get the following special cases.

\subsection{Case 1: fractional heat equation}

If we take $a=0$ in Eq. (1.1), we have the following fractional diffusion equation:

$$
{ }_{0}^{A B C} D_{t}^{\alpha} \mathcal{U}(x, t)=\frac{\partial^{2} \mathcal{U}(x, t)}{\partial x^{2}}, \quad 0<\alpha \leq 1
$$

with

$$
\phi(x)=\sin (\pi x),
$$

On comparing with Eq. (4.3), and using Eq. (5.4) we get

$$
N[\rho(x, t, q)]=\frac{1}{p}(\sin \pi x)+\left[\frac{1-\alpha}{\mathcal{B}(\alpha)}\left(1+\frac{\alpha}{(1-\alpha) p^{\alpha}}\right)\right] L\left\{\frac{\partial^{2} \sigma(x, t: q)}{\partial x^{2}}\right\}
$$

using $q$-HATM, which is discussed in Sect. 3, we get

$$
\begin{aligned}
\mathcal{U}_{0}(x, t)= & \sin (\pi x), \\
\mathcal{U}_{1}(x, t)= & h \pi^{2}(\sin \pi x)\left(\frac{1-\alpha}{B(\alpha)}\right)\left[1+\frac{\alpha}{(1-\alpha)} \frac{t^{\alpha}}{\Gamma(\alpha+1)}\right], \\
\mathcal{U}_{2}(x, t)= & (n+h) u_{1}+h^{2} \pi^{4}(\sin \pi x)\left(\frac{1-\alpha}{B(\alpha)}\right)^{2} \\
& \times\left[1+\frac{2 \alpha t^{\alpha}}{(1-\alpha) \Gamma(\alpha+1)}+\left(\frac{\alpha}{1-\alpha}\right)^{2} \frac{t^{2 \alpha}}{\Gamma(2 \alpha+1)}\right],
\end{aligned}
$$

By substituting the values obtained from Eqs. (6.3), (6.4), (6.5), ... in Eq. (5.14), we obtain the solution of fractional heat equation.

\subsection{Case 2: slow diffusion processes of fractional order}

Taking $a=2$, we get

$$
{ }_{0}^{A B C} D_{t}^{\alpha} \mathcal{U}(x, t)=\frac{\partial}{\partial x}\left(\mathcal{U}^{2} \frac{\partial \mathcal{U}(x, t)}{\partial x}\right)
$$

known as a slow diffusion equation, having the initial condition

$$
\phi(x)=\frac{x+b}{2 c} .
$$

By $q$-HATM, we get the respective successive terms:

$$
\mathcal{U}_{0}(x, t)=\frac{x+b}{2 c},
$$




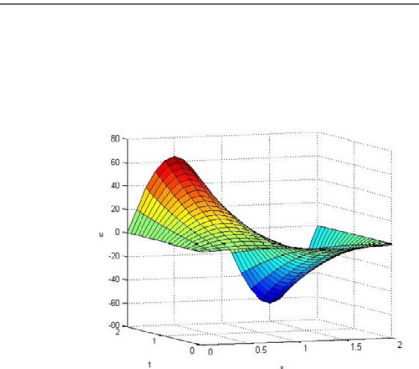

\section{CASE 1}
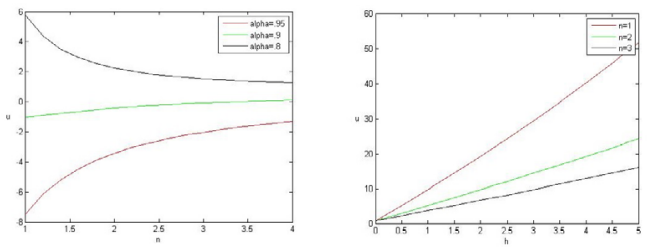

(A) $\mathcal{U}(x, t)$ w.r.t.

(B) $\mathcal{U}(x, t)$ w.r.t. $n($ с) $\mathcal{U}(x, t)$ w.r.t. $h$ $x$ and $t$,when $\alpha=1$, $h=-1, n=1$, for different values for different values of $\alpha$, when $h=$ of $n$, when $\alpha=1, x=$ $-1, x=0.3, t=0.3 \quad 0.3, t=0.3$
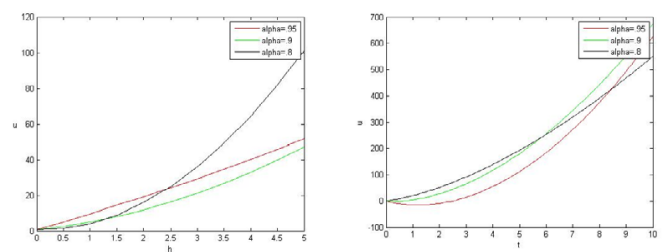

(D) $\mathcal{U}(x, t)$ w.r.t. $h(\mathrm{E}) \mathcal{U}(x, t)$ w.r.t. $t$ for different values for different values of $\alpha$, when $n=$ of $\alpha$, when $n=$ $1, x=0.3, t=0.3 \quad 1, h=-1, x=0.3$

Figure 1 Results of Case 1

$$
\begin{aligned}
\mathcal{U}_{1}(x, t)= & -h\left(\frac{x+b}{4 c^{3}}\right)\left(\frac{1-\alpha}{B(\alpha)}\right)\left[1+\frac{\alpha}{(1-\alpha) \Gamma(\alpha+1)} t^{\alpha}\right], \\
\mathcal{U}_{2}(x, t)= & (n+h) u_{1}+3 h^{2} \frac{(x+b)}{8 c^{5}}\left(\frac{1-\alpha}{B(\alpha)}\right)^{2} \\
& \times\left[1+\frac{2 \alpha t^{\alpha}}{(1-\alpha) \Gamma(\alpha+1)}+\left(\frac{\alpha}{1-\alpha}\right)^{2} \frac{t^{2 \alpha}}{\Gamma(2 \alpha+1)}\right],
\end{aligned}
$$

By substituting the values obtained from Eqs. (6.8), (6.9), (6.10), ... in Eq. (5.14), we obtain the solution.

\subsection{Case 3: fast diffusion equation of fractional order}

If we take $a=-2$ in Eq. (1.1), we have following equation:

$$
{ }_{0}^{A B C} D_{t}^{\alpha} \mathcal{U}(x, t)=\frac{\partial}{\partial x}\left(\mathcal{U}^{-2} \frac{\partial \mathcal{U}(x, t)}{\partial x}\right),
$$

known as the fractional fast diffusion equation with

$$
\phi(x)=\frac{1}{\sqrt{\left(1+x^{2}\right)}} .
$$




\section{CASE 2}
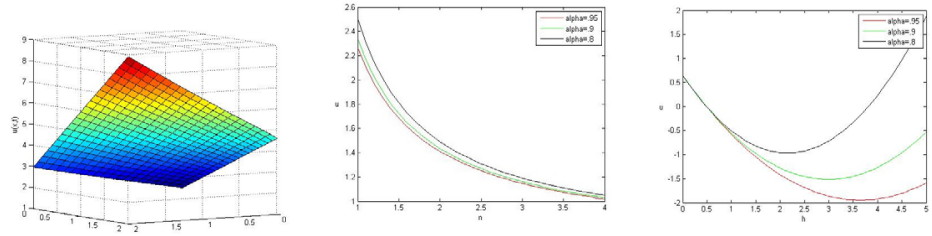

(A) $\mathcal{U}(x, t)$ w.r.t. (B) $\mathcal{U}(x, t)$ w.r.t. $n($ C) $\mathcal{U}(x, t)$ w.r.t. $h$ $x$ and $t$, when $\alpha=1$ for different values for different values $h=-1, n=1, b=$ $1, c=1$ of $\alpha$ when $h=$ of $\alpha$ when $h=$ $-1, x=t=-1, n=1, x=t=$ $0.3, b=c=1 \quad .3, b=c=1$
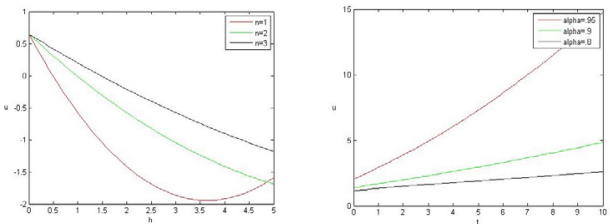

(D) $\mathcal{U}(x, t)$ w.r.t. (E) $\mathcal{U}(x, t)$ w.r.t. $t$ $h$ for different val- for different values ues of $n$, when of $\alpha$ when $h=$ $\alpha=1, x=0.3, t=-1, n=1, x=t=$ $0.3, b=c=1 \quad .3, b=c=1$

Figure 2 Results of Case 2

By the help of $q$-HATM, we found the following iterative terms:

$$
\begin{aligned}
\mathcal{U}_{0}(x, t)= & \left(1+x^{2}\right)^{-1 / 2}, \\
\mathcal{U}_{1}(x, t)= & -h\left(\frac{1-\alpha}{B(\alpha)}\right)\left[1+\frac{\alpha}{(1-\alpha)} \frac{t^{\alpha}}{\Gamma(\alpha+1)}\right]\left[\frac{-1}{\left(1+x^{2}\right)^{3 / 2}}\right], \\
u_{2}(y, t)= & (n+h) u_{1}-h^{2}\left(\frac{1-\alpha}{B(\alpha)}\right)^{2}\left(\frac{1-2 x^{2}}{\left(1+x^{2}\right)^{5 / 2}}\right) \\
& \times\left[1+\frac{2 \alpha t^{\alpha}}{(1-\alpha) \Gamma(\alpha+1)}+\left(\frac{\alpha}{1-\alpha}\right)^{2} \frac{t^{2 \alpha}}{\Gamma(2 \alpha+1)}\right],
\end{aligned}
$$

By substituting the values obtained from Eqs. (6.14), (6.15), ... in Eq. (5.14) we obtain the approximate result of the above problem.

Remark In this method, we have a free hand to give certain values to $h$ and $n$, which helps in the convergence of the result. 


\section{CASE 3}
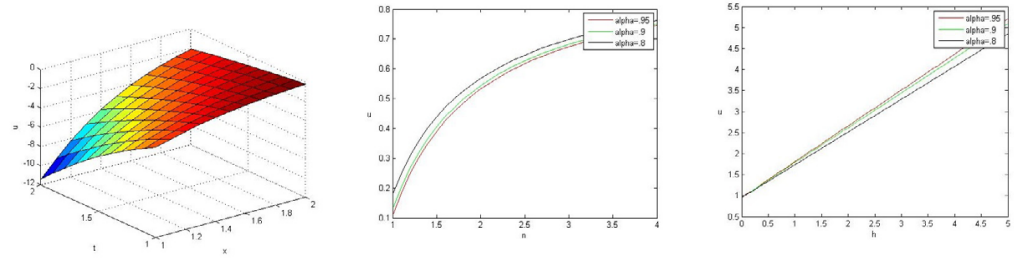

(B) $\mathcal{U}(x, t)$ w.r.t. $n($ c) $\mathcal{U}(x, t)$ w.r.t. $h$ (A) $\mathcal{U}(x, t)$ w.r.t. $x$ and $t$, when $\alpha=1$, $h=-1, n=1$ for different values for different values of $\alpha$, when $h=$ of $\alpha$, when $n=$ $-1, x=0.3, t=0.3 \quad 1, x=0.3, t=0.3$
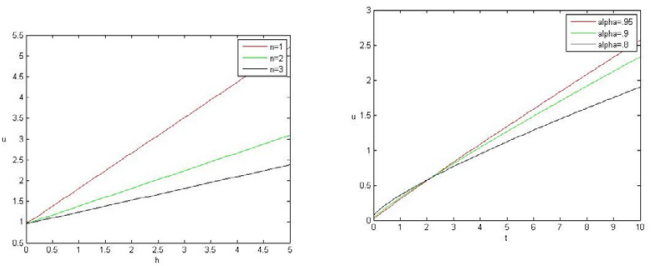

(D) $\mathcal{U}(x, t)$ w.r.t. $h$ (E) $\mathcal{U}(x, t)$ w.r.t. $t$ for different values for different values of $n$, when $\alpha=1, x=$ of $\alpha$, when $h=$ $0.3, t=0.3$ $-1, n=1, x=t=$ 0.3

Figure 3 Results of Case 3

\section{Conclusion}

Besides the mathematical satisfactions of the Atangana-Baleanu derivative with fractional order, the focus on this derivative is because of the necessity of employing a model portraying the behavior of orthodox viscoelastic materials, thermal medium and others. The approximate solution of nonlinear fractional diffusion equations is obtained using a $q$ homotopy analysis transform method. Further, we discuss the effect of various values of $\alpha, h, n$ in the solution. These results are given in the form of a graphical representation (see Figs. 1(a)-(e) to 5 for Case 1, Figs. 2(a)-(e) for Case 2 and Figs. 3(a)-(e) for Case 3). The method indicates that, for various values $n$ and $h$, the solution converges. In our future work, we can compare the applicability of various fractional derivatives on this equation and can try to compare their solutions as discussed in [33]

\section{Acknowledgements}

The author sincerely thanks the reviewers for their valuable comments.

\section{Funding}

The author would like to extend his sincere appreciation to the Deanship of Scientific Research at King Saud University for funding this group No. RG-1440-025.

Availability of data and materials

There is no data used in the paper. 
Authors' contributions

The author contributed to writing the draft and the software and reviewed and approved the final version of the manuscript.

\section{Publisher's Note}

Springer Nature remains neutral with regard to jurisdictional claims in published maps and institutional affiliations.

Received: 14 April 2021 Accepted: 10 June 2021 Published online: 10 July 2021

\section{References}

1. Vazquez, J.L.: Lecture Notes in Mathematics Springer, Berlin (2017)

2. Wu, Z., Yin, J., Li, H., Zhao, J.: World Scientific, Singapore, (2001)

3. Miller, K.S., Ross, B.: An Introduction to the Fractional Calculus and Fractional Differential Equations. Wiley, New York (1993)

4. Podlubny, I.: Fractional Differential Equations. Academic Press, New York (1999)

5. Kilbas, A.A., Srivastava, H.M., Trujillo, J.J.: Theory and Applications of Fractional Differential Equations. Elsevier, Amsterdam (2006)

6. Hilfer, R. (ed.): Applications of Fractional Calculus in Physics World Scientific, Singapore (2000)

7. Chaurasia, V.B.L., Dubey, R.S., Belgacem, F.B.M.: Fractional radial diffusion equation analytical solution via Hankel and Sumudu transforms. Math. Eng. Sci. Aerosp. 3, 1-10 (2012)

8. Caputo, M., Fabrizio, M.: Prog. Fract. Differ. Appl. 1(2), 73-85 (2015)

9. Dubey, R.S., Goswami, P., Belgacem, F.B.M.: Generalized time-fractional telegraph equation analytical solution by Sumudu and Fourier transforms. J. Fract. Calc. Appl. 5, 52-58 (2014)

10. Yang, X.J., Machado, J.A.T., Cattani, C., Gao, F.: On a fractal LC-electric circuit modeled by local fractional calculus. Commun. Nonlinear Sci. Numer. Simul. 47, 200-206 (2017)

11. Debbouche, A., Nieto, J.J.: Approximate controllability of fractional delay dynamic inclusions with nonlocal control conditions. Appl. Math. Comput. 243, 161-175 (2014)

12. Alkahtani, B.S.T., Alkahtani, O.J., Dubey, R.S., Goswami, P.: Solution of fractional oxygen diffusion problem having without singular kernel. J. Nonlinear Sci. Appl. 11, 1-9 (2016)

13. Alkahtani, B.S.T., Alkahtani, O.J., Dubey, R.S., Goswami, P.: The solution of modified fractional Bergman's minimal blood glucose-insulin model. Entropy 19(5), Article ID 114 (2017)

14. Hristov, J.: An approximate analytical (integral-balance) solution to a non-linear heat diffusion equation. Therm. Sci. 19(2), $723-733(2015)$

15. Yang, X.-J., Hristov, J., Srivastava, H.M., Ahmad, B.: Modelling fractal waves on shallow water surfaces via local fractional Korteweg-de Vries equation. Abstr. Appl. Anal. 2014, Article ID 278672 (2014)

16. Hristov, J.: Transient heat diffusion with a non-singular fading memory from the Cattaneo constitutive equation with Jeffrey's kernel to the Caputo-Fabrizio time-fractional derivative. Therm. Sci. 20(2), 757-762 (2016)

17. Jain, R., Arekar, K., Dubey, R.S.: Study of Bergman's minimal blood glucose-insulin model by Adomian decomposition method. J. Inf. Optim. Sci. 38(1), 133-149 (2017)

18. Dubey, R.S., Alkahtani, B.S.T., Atangana, A.: Analytical solution of space-time fractional Fokker Plank equation by homotopy perturbation Sumudu transform method. Math. Probl. Eng. 2015, Article ID 780929 (2015)

19. Almuqrin, M.A., Goswami, P., Sharma, S., Khan, I., Dubey, R.S., Khan, A.: Fractional Model of Ebola Virus In Population of Bats In Frame of Atangana-Baleanu Fractional Derivative, Results Phys. (in press)

20. Atangana, A.: Fractional Operators with Constant and Variable Order with Application to Geo-Hydrology. Academic Press, New York (2018)

21. Alkahtani, B.S.T.: Chua's circuit model with Atangana-Baleanu derivative with fractional order. Chaos Solitons Fractals 89, 547-551 (2016)

22. Gómez-Aguilar, J.F., Atangana, A.: Electrical circuits RC, LC, and RL described by Atangana-Baleanu fractional derivatives. Int. J. Circuit Theory Appl. 45(11), 1514-1533 (2017)

23. Koca, I., Atangana, A.: Solutions of Cattaneo-Hristov model of elastic heat diffusion with Caputo-Fabrizio and Atangana-Baleanu fractional derivatives. Therm. Sci. 21(6), 2299-2305 (2017)

24. Atangana, A., Baleanu, D.: New fractional derivatives with nonlocal and nonsingular kernel: theory and application to heat transfer model. Therm. Sci. 20(2), 763-769 (2016)

25. Atangana, A.: On the new fractional derivative and application to nonlinear Fisher's reaction-diffusion equation. Appl. Math. Comput. 273, 948-956 (2016)

26. Atangana, A., Baleanu, D.: New fractional derivatives with nonlocal and non-singular kernel: theory and application to heat transfer model. Therm. Sci. 20, 763 (2016)

27. Tateishi, A.A., Ribeiro2, H.V., Lenzi, E.K.: Front. Phys. 5, 52 (2017)

28. Liao, S.: Beyond Perturbation: Introduction to Homotopy Analysis Method. CRC Press, Boca Raton (2000)

29. Liao, S.J.: Notes on the homotopy analysis method: some definitions and theorems. Commun. Nonlinear Sci. Numer Simul. 14(9), 83-97 (2009)

30. El-Tawil, M.A., Huseen, S.N.: The q-homotopy analysis method (q-HAM). Int. J. Appl. Math. Mech. 8, 51-75 (2012)

31. Wazwaz, A.M., El-Sayed, S.M.: A new modification of the Adomian decomposition method for linear and nonlinear operators. Appl. Math. Comput. 122(3), 393-405 (2001)

32. Dubey, R.S., Goswami, P.: Analytical solution of the nonlinear diffusion equation. Eur. Phys. J. Plus 133(5), Article ID 183 (2018)

33. Malyk, I., Mohammed, M., Shrahili, A., Shafay, A.R., Goswami, P., Sharma, S., Dubey, R.S.: Analytical solution of non-linear fractional Burger's equation in the framework of different fractional derivative operators. Results Phys. 19, Article ID $103397(2020)$

34. Kilicman, A., Shokhanda, R., Goswami, P.: On the solution of $(n+1)$-dimensional fractional M-Burgers equation. Alex Eng. J. 60(1), 1165-1172 (2021) 
35. Adomian, G.: A review of the decomposition method in applied mathematics. J. Math. Anal. Appl. 135, 501-544 (1988)

36. He, H.-J.: Homotopy perturbation technique. Comput. Math. Appl. Mech. Eng. 178, 257-262 (1999)

37. Atangana, A., Nieto, J.J.: Numerical solution for the model of RLC circuit via the fractional derivative without singular kernel. Adv. Mech. Eng. 7(10), 1-7 (2015)

38. Caputo, M.: Elasticita e Dissipazione. Zanichelli, Bologna (1969)

Submit your manuscript to a SpringerOpen ${ }^{\circ}$ journal and benefit from:

- Convenient online submission

- Rigorous peer review

- Open access: articles freely available online

- High visibility within the field

- Retaining the copyright to your article

Submit your next manuscript at $\gg$ springeropen.com 